\title{
OPEN Association of dietary approaches to stop hypertension eating style and risk of sarcopenia
}

\author{
Sanaz Soltani ${ }^{1}$, Rezvan Hashemi ${ }^{2 凶}$, Ramin Heshmat ${ }^{3}$, Ahmadreza Dorosty Motlagh $^{1}$ \& \\ Ahmad Esmaillzadeh ${ }^{1,4,5} \bowtie$
}

The association between habitual intake of the "dietary approaches to stop hypertension" (DASH) eating plan and sarcopenia has received limited attention. The present study aimed to investigate the association between adherence to DASH dietary pattern and sarcopenia and its components including muscle mass, muscle strength, and muscle performance among community-dwelling older adults population. This population-based cross-sectional study was performed in 2011 among 300 older people ( 150 men and 150 women) aged $\geq 55$ years, who were selected using cluster random sampling method. Dietary intake of study participants were examined by the use of a Block-format 117-item food frequency questionnaire (FFO). The DASH score was constructed based on eight main foods and nutrients emphasized or minimized in the DASH diet. All components of sarcopenia was measured using standard protocols and sarcopenia was defined based on both former and new European Working Group on Sarcopenia in Older People (EWGSOP) guidelines. Mean age and BMI of study participants were $66.7 \pm 7.7$ years and $27.3 \pm 4.2 \mathrm{~kg} / \mathrm{m}^{2}$, respectively. Totally, 31 individuals meet the criteria of EWGSOP2-sarcopenia. We found no significant association between adherence to the DASH diet and EWGSOP2-sarcopenia either before (OR 1.08; 95\% Cl 0.45-2.54) or after adjustment for potential confounders $(\mathrm{OR} 1.04 ; 95 \% \mathrm{Cl} 0.39-2.75)$. The same findings were obtained in the gender-stratified analyses (men: OR 2.29; 95\% Cl 0.39-13.29 and women: $0.75 ; 95 \% \mathrm{Cl} 0.23-2.45$ ). In conclusion, we found that adherence to the DASH-style diet was not significantly associated with odds of sarcopenia. Future prospective studies are required to confirm these findings.

Sarcopenia as a geriatric syndrome ${ }^{1}$ is a concept that describes the loss of skeletal muscle mass and strength ${ }^{2}$. This condition is associated with the risk of physical frailty, functional impairment, poor quality of life, and premature death in older individuals ${ }^{3,4}$. Interest in sarcopenia identification has grown since its recent recognition as a muscle disease with an International Classification of Diseases, Tenth Revision, Clinical Modification (ICD-10-CM) diagnosis code 5 . Sarcopenia wastes a significant cost in the healthcare systems. In 2000, about $\$ 18.5$ billion (1.5\% of total healthcare expenditures) was spent in the United States on healthcare expenses related to sarcopenia and its complications ${ }^{6}$. In Iran, there is no information on the costs that sarcopenia imposes on the healthcare system. The prevalence of this disorder varies largely based on the definition or cut-off value across studies $^{7}$. Accordingly, the prevalence of sarcopenia varies worldwide from 9.9 to $40.4 \%{ }^{8}$.

Healthy eating is highly recommended to preserve physical health in later life. Poor diets and nutritional status are common among older people ${ }^{9,10}$. The Dietary Approaches to Stop Hypertension (DASH) eating plan, as a healthy dietary pattern, characterized by low intake of saturated fat, meats, sweets, added sugars, and sugarsweetened beverages and high intakes of fruit and vegetables, low-fat dairy, whole-grain products, nuts, fish and poultry ${ }^{11}$. This dietary pattern has been protectively linked with several conditions such as cardiovascular disease $^{12}$, cancer $^{13}$, type 2 diabetes ${ }^{14}$; however, limited data are available examining adherence to this dietary pattern in relation to sarcopenia. Although usual nutritional advices to prevent sarcopenia include protein or amino acids intake, the DASH diet, through its myo-protective components ${ }^{15-19}$, might also play a direct or indirect

\footnotetext{
${ }^{1}$ Department of Community Nutrition, School of Nutritional Sciences and Dietetics, Tehran University of Medical Sciences, P.O. Box 14155-6117, Tehran, Iran. 'Department of Geriatric Medicine, Ziaeian Hospital, Tehran University of Medical Sciences, Tehran, Iran. ${ }^{3}$ Chronic Diseases Research Center (CDRC), Endocrinology and Metabolism Population Sciences Institute, Tehran University of Medical Sciences, Tehran, Iran. ${ }^{4}$ Obesity and Eating Habits Research Center, Endocrinology and Metabolism Molecular-Cellular Sciences Institute, Tehran University of Medical Sciences, Tehran, Iran. ${ }^{5}$ Department of Community Nutrition, Isfahan University of Medical Sciences,

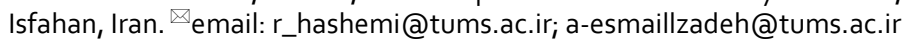


role in maintaining muscle health. Adherence to DASH diet may directly affect muscle health by ameliorating metabolic processes (like oxidative stress, inflammation and insulin resistance) involved in sarcopenia ${ }^{20-27}$. In addition, DASH diet may reduce the risk of age-related chronic conditions (e.g., cardiovascular diseases, diabetes and cancer), that are associated with worsening muscle health ${ }^{12,14,28-31}$. We are aware of only two studies that examined the link between DASH diet and sarcopenia or its component ${ }^{32,33}$. Adherence to this eating style in African American and White adults was associated with better handgrip strength (HS), which is a simple and reliable method to evaluate muscle function ${ }^{32}$. However, the study of Kuczmarski et al. examined the association of DASH diet with handgrip strength alone, not the sarcopenia ${ }^{32}$. In a quasi-experimental study by Straight et al., administration of DASH diet for eight weeks in overweight and obese older adults resulted in improved body composition, muscle strength, and physical function ${ }^{33}$. Although findings from both observational and interventional studies are needed to establish the association between DASH diet and sarcopenia, findings from interventional studies cannot easily be extrapolated to human routine life. Interventional studies might be appropriate to detect the effect of an intervention on sarcopenic conditions. In order to assess if adherence to the DASH diet can prevent sarcopenia, it seems that observational studies are required. Therefore the present study aimed to investigate the association between adherence to DASH dietary pattern and sarcopenia and its components including muscle mass, muscle strength, and muscle performance among community-dwelling older adults population.

\section{Materials and methods}

Participants. This population-based cross-sectional study was performed from May to October of 2011 in Tehran, Iran (the detailed report on the sampling method and data collection procedure, have been published previously ${ }^{34}$ ). We enrolled 300 older people ( 150 men and 150 women) aged $\geq 55$ years from district 6 of Tehran using cluster random sampling method. We selected the head of each 30 cluster based on a ten-digit postal code and enrolled individuals aged $\geq 55$ years, with the ability to move without crutches, walker or assistive devices and those without any active cancers (based on self-reported data). We did not include people who were susceptible to sarcopenia including individuals with artificial limbs or limb prosthesis and those with a history of debilitating disease including Congestive Heart Failure (CHF), Chronic Obstructive Pulmonary Disorder (COPD), Chronic Renal Failure (CRF), cirrhosis and liver failure (based on self-reported data).

The study was conducted according to the guidelines of Declaration of Helsinki. The study protocol was approved by the Tehran University of Medical Sciences ethics committee. At first, participants were briefly informed about the objectives of the survey. All participants completed the written informed consent before data collection. Then all required data were collected through face-to-face method by a trained interviewer at home.

Dietary assessments. Dietary intake of study participants were examined by the use of a Block-format 117-item food frequency questionnaire (FFQ). The questionnaire included a list of food items, along with a given portion size and an open-ended frequency response section. A trained nutritionist administered the FFQs. Participants were requested to report their daily, weekly or monthly frequency consumption of each food item in the questionnaire during the preceding year. Frequency data in the FFQ were then converted to grams per day considering the household measures of portion sizes. Then these data were linked to the modified food composition database of the US Department of Agriculture, using Nutritionist IV software (version 7.0; N-Squared Computing, Salem, OR, USA), to compute daily energy and nutrients intake of each participant. The seasonal variation in dietary intake of fruit and vegetables was considered in the questionnaire; such that participants were requested to report their consumption of specific fruit and vegetables during the time of availability on that item. Then, when we were computing the grams of food items, we considered duration of availability of that food item in a year in our calculation.

Although the FFQ was validated previously ${ }^{35}$, we conducted a pilot study on 30 participants to examine its validity in older adults' population. To do this, nutrient intakes of the FFQ were compared with those obtained from four dietary records (two records in weekdays and two other records after 2 months). The results showed a good correlation between the dietary intakes determined by the FFQ and those from the self-reported records. The correlation coefficient for animal protein, fruits, and vegetables were $0.43,0.57$, and 0.45 , respectively. The energy adjusted correlation coefficients for $\beta$-carotene and vitamin $C$ were 0.65 and 0.76 , respectively.

Adherence to DASH-style diet. In this study, to indicate the degree of adherence to DASH-style diet, we constructed DASH scores based on foods and nutrients emphasized or minimized in the DASH diet, focusing on eight components: high intake of fruits, vegetables, nuts and legumes, low-fat dairy products, and whole grains and low intake of sodium, sugar-sweetened beverages (SSB) and sweets, and red and processed meats. For each participant, energy-adjusted intakes of the above-mentioned foods and nutrients were calculated using residual method $^{36}$. Then, participants were categorized into deciles of the energy-adjusted intakes of foods and nutrients. Subjects in the highest decile of fruits, vegetables, whole grains, low-fat dairy products, nuts, legumes and soy were assigned the score of 10 and those in the lowest decile were assigned the score of 1 . With regards to red and processed meat, sugar-sweetened beverages (SSB) and sweets, and sodium, we did vice versa; that is individuals in the highest decile were assigned the score of 1 and those in the lowest decile were assigned the score of 10. Then, the overall DASH score was calculated by summing up the scores of eight components. Therefore, each participant has a score between 8 and 80 .

Assessment of sarcopenia. We applied both former ${ }^{37}$ and new ${ }^{38}$ European Working Group on Sarcopenia in Older People (EWGSOP) guidelines for sarcopenia in our study. EWGSOP1 recommends considering the combination of both low muscle mass and low muscle function (either strength or performance) in 
the definition ${ }^{37}$. Based on EWGSOP2, low muscle strength is a key component of sarcopenia. In addition, this definition uses low muscle quantity and quality to confirm the diagnosis, and considers poor physical performance as indicative of severe sarcopenia ${ }^{38}$. The muscle mass was measured as the ratio of an individual's total lean mass of legs and arms (also named Appendicular Skeletal Muscle or ASM) ${ }^{39}$ to their squared height (ASM/ height ${ }^{2}$ ). A DXA scanner (Discovery model, manufactured by Hologic, Inc. Bedford USA: W S/N 84430) was used to measure ASM. The coefficients of variation of the DEXA for fat and lean mass were $1.5 \%$ and $0.6 \%$, respectively. According to EWGSOP1, low muscle mass was defined as the amount of less than $5.45\left(\mathrm{~kg} / \mathrm{m}^{2}\right)$ and $7.26\left(\mathrm{~kg} / \mathrm{m}^{2}\right)$ for women and men, respectively ${ }^{37}$. These cut-off points were changed to less than $5.5(\mathrm{~kg} /$ $\left.\mathrm{m}^{2}\right)$ and $7.0\left(\mathrm{~kg} / \mathrm{m}^{2}\right)$ for women and men, respectively ${ }^{38}$. A handgrip test by a pneumatic instrument-a squeeze bulb dynamometer (manufactured by Jamar,Inc. USA: c7489-02 Rolyan) calibrated in pound per square inch (psi) - was used to scale the muscle strength. The position of the hands while doing the hand-grip strength was based on the recommendations of the American Society of Hand Therapists ${ }^{40}$. To do this, we asked the patient to sit in a straight-backed chair while shoulders adducted in neutral, arms unsupported and elbows flexed at $90^{\circ}$. At this position, forearm rotation was neutral and wrist $0^{\circ}-30^{\circ}$ dorsiflexion and $0^{\circ}-15^{\circ}$ ulnar deviated. The hand grip strength (maximum voluntary contractions) was measured three times for each right and left hand with a 30 -s rest in between measurements. We used the average measurements of the participants' both hands as their muscle strength. Sex and age-specific cutoff points suggested by Merkies et al. were used to identify low muscle strength $^{41}$. These values were different from EWGSOP1 cutoff points, in which hand grip strength $<30 \mathrm{~kg}$ for men and $<20 \mathrm{~kg}$ for women was considered as low grip strength ${ }^{37}$. In the revised definition, these cut-off points were changed to $<27 \mathrm{~kg}$ for men and $<16 \mathrm{~kg}$ for women ${ }^{38}$. The muscle performance was measured using a $4-\mathrm{m}$ walk gait speed test ${ }^{37}$. Participants who had gait speeds less than $0.8 \mathrm{~m} / \mathrm{s}$ were identified as those with a low muscle performance ${ }^{37}$.

Assessment of other variables. General information on age, sex, socio-economic status, medical history, medication use, smoking habits and alcohol consumption was collected by a trained dietitian. Physical activity level was examined by the use of a short form of International Physical Activity Questionnaire (IPAQ). The validity of IPAQ has previously been examined in elderly population ${ }^{35}$. Vigorous- and moderate-intensity activities and walking (for at least $10 \mathrm{~min}$ ) were asked separately in minutes and days during the past week. Then MET scores for each activity were obtained from earlier publications ${ }^{42}$. The obtained MET scores were multiplied by the amount of time each participant spent on that activity, while taking into consideration the frequency of engaging in the mentioned activity during the past week. Then, the scores for different activities were summed up to obtain total MET-min/week. Weight was measured using a digital scale while participants were minimally clothed. A wall tape measure was used to assess height in standing position without shoes. Participants were asked to stand up and normally breathe to measure waist circumference at the middle of lower rib margin and iliac crest. Weight $(\mathrm{kg})$ divided by height squared $\left(\mathrm{m}^{2}\right)$ was used to calculate body mass index (BMI).

Statistical analysis. Participants were classified based on tertiles of DASH dietary pattern scores. To compare general characteristics of study participants across tertiles of DASH dietary pattern scores, we used one-way analysis of variance and chi-squared tests, where appropriate. Dietary intakes were compared using ANCOVA, adjusting for the confounding effects of age, sex and energy intake. To find the association between DASH dietary pattern and risk of sarcopenia, we used multivariate logistic regression analysis. In the first statistical model, we controlled for age (years), sex (male/female) and energy intake (kcal/day). Further adjustment was applied for physical activity (MET-h/week), smoking (yes/no), medication use (yes/no), alcohol consumption (yes/no), and positive history of disease (yes/no) in the second model. To assess the trend of odds ratios across categories of tertiles of DASH diet score, we considered the tertile categories as an ordinal variable in the logistic regression models. All statistical analyses were conducted using SPSS, version 18 (SPSS Inc., Chicago, IL, USA). $P$ values less than 0.05 were considered statistically significant.

\section{Results}

General characteristics of study participants in subjects with and without sarcopenia as well as across tertiles of DASH diet score are displayed in Table 1. Patients with EWGSOP2-sarcopenia had lower BMI and diabetes and used more sexual hormone and corticosteroids than subjects without sarcopenia. Individuals in the highest tertile of DASH diet score were more likely to be female and less likely to be smoker and alcohol user, compared with those in the lowest tertile. No other significant differences were found.

Multivariable-adjusted dietary intakes of study participants across tertiles of DASH diet score are provided in Table 2. Participants in the top tertile of DASH diet score had significantly higher intakes of dairy products, fruits, vegetables, nuts, legumes and soy, dietary fiber, protein, calcium and folate and lower intakes of sugar-sweetened beverages and sweets, red and processed meats, energy, sodium and fat compared with those in the bottom tertile.

The prevalence of EWGSOP2-sarcopenia and its components across tertile categories of DASH diet score as well as stratified by gender are indicated in Table 3. In the whole population, muscle mass and hand grip strength were significantly lower among subjects in the highest tertile of DASH diet score compared with those in the lowest tertile. The prevalence of EWGSOP2-sarcopenia itself and its other components were not significantly different across tertiles of DASH diet score. When we did gender-stratified analyses, no significant differences were found across tertiles of DASH diet score, in both men and women.

Multivariable-adjusted odds ratios for sarcopenia across tertile categories of DASH diet score as well as stratified by gender are provided in Table 4 . We found no significant association between adherence to the DASH diet and EWGSOP2-sarcopenia (OR 1.08; 95\% CI 0.45-2.54). Such finding was also seen when we adjusted the ORs for potential confounders (OR 1.04; 95\% CI 0.39-2.75). The same findings were obtained in the gender-stratified 


\begin{tabular}{|c|c|c|c|c|c|c|c|}
\hline & \multicolumn{2}{|l|}{ Sarcopenia $^{\dagger}$} & \multirow[b]{2}{*}{$p^{\ddagger}$} & \multicolumn{3}{|c|}{ Tertiles of DASH diet score } & \multirow[b]{2}{*}{$P^{\ddagger}$} \\
\hline & Yes $(n=31)$ & No $(n=269)$ & & $T_{1}(n=96)$ & $T_{2}(n=98)$ & $T_{3}(n=106)$ & \\
\hline Age (years) & $64.54 \pm 6.39$ & $67.05 \pm 7.81$ & 0.08 & $66.09 \pm 7.81$ & $66.50 \pm 7.58$ & $67.70 \pm 7.71$ & 0.29 \\
\hline BMI $\left(\mathrm{kg} / \mathrm{m}^{2}\right)$ & $23.48 \pm 2.78$ & $27.83 \pm 4.11$ & $<0.001$ & $27.31 \pm 4.08$ & $27.69 \pm 4.16$ & $27.15 \pm 4.37$ & 0.65 \\
\hline $\begin{array}{l}\text { Physical activity } \\
\text { (MET-h/w) }\end{array}$ & $1223.45 \pm 1122.32$ & $1302.72 \pm 1462.33$ & 0.77 & $1187.69 \pm 1262.57$ & $1357.44 \pm 1606.94$ & $1333.11 \pm 1405.76$ & 0.67 \\
\hline Female (\%) & 64.5 & 49.4 & 0.11 & 40.6 & 49.0 & 62.3 & 0.008 \\
\hline Alcohol use (\%) & 9.7 & 13.8 & 0.52 & 18.8 & 19.4 & 2.8 & $<0.001$ \\
\hline Smoking (\%) & 12.9 & 12.6 & 0.96 & 25.0 & 9.2 & 4.7 & $<0.001$ \\
\hline \multicolumn{8}{|l|}{ Medical history } \\
\hline Diabetes (\%) & 6.5 & 22.3 & 0.03 & 16.7 & 24.5 & 20.8 & 0.40 \\
\hline MI (\%) & 6.5 & 12.6 & 0.31 & 12.5 & 11.2 & 12.3 & 0.95 \\
\hline CVA (\%) & 6.5 & 2.2 & 0.16 & 4.2 & 3.1 & 0.9 & 0.34 \\
\hline Arthritis (\%) & 3.2 & 1.5 & 0.47 & 2.1 & 2.0 & 0.9 & 0.77 \\
\hline Asthma (\%) & 3.2 & 1.9 & 0.60 & 2.1 & 1.0 & 2.8 & 0.65 \\
\hline \multicolumn{8}{|l|}{ Drug history } \\
\hline $\begin{array}{l}\text { Sexual hormone } \\
\text { use (\%) }\end{array}$ & 9.7 & 2.2 & 0.02 & 2.1 & 2.0 & 4.7 & 0.43 \\
\hline Statin use (\%) & 35.5 & 36.8 & 0.88 & 33.3 & \begin{tabular}{|l|}
38.8 \\
\end{tabular} & 37.7 & 0.70 \\
\hline ACEi use $(\%)^{\S}$ & 3.2 & 8.2 & 0.32 & 7.3 & 7.1 & 8.5 & 0.92 \\
\hline $\begin{array}{l}\text { Corticosteroid } \\
\text { use (\%) }\end{array}$ & 12.9 & 1.5 & $<0.001$ & 3.1 & 2.0 & 2.8 & 0.88 \\
\hline
\end{tabular}

Table 1. Characteristics of study participants in subjects with and without sarcopenia and also in tertile categories of DASH diet score. All values are mean \pm SD, unless indicated. ${ }^{\dagger}$ Sarcopenia was defined based on European Working Group on Sarcopenia in Older People 2 (EWGSOP2) definition ${ }^{38}$. ${ }^{\ddagger}$ ANOVA for continuous variables and Chi-squared test for categorical variables. ${ }^{\S} \mathrm{ACEi}$ : angiotensin-converting enzyme inhibitor.

\begin{tabular}{|c|c|c|c|c|}
\hline & \multicolumn{3}{|c|}{ Tertiles of DASH diet score } & \multirow[b]{2}{*}{$\boldsymbol{P}^{\dagger}$} \\
\hline & $T_{1}(n=96)$ & $T_{2}(n=98)$ & $T_{3}(n=106)$ & \\
\hline DASH score range & $<39$ & $39-49$ & $>49$ & \\
\hline \multicolumn{5}{|l|}{ Food groups (g/day) } \\
\hline Fruits & $455.76 \pm 24.07$ & $629.17 \pm 23.76$ & $717.97 \pm 22.94$ & $<0.001$ \\
\hline Vegetables & $404.88 \pm 22.73$ & $536.36 \pm 22.44$ & $709.17 \pm 21.66$ & $<0.001$ \\
\hline Nuts, legumes and soy & $53.71 \pm 3.70$ & $55.64 \pm 3.66$ & $67.13 \pm 3.53$ & 0.01 \\
\hline Dairy products & $498.26 \pm 30.83$ & $544.11 \pm 30.45$ & $628.61 \pm 29.39$ & 0.009 \\
\hline Grains & $333.29 \pm 18.09$ & $307.94 \pm 17.87$ & $307.34 \pm 17.24$ & 0.50 \\
\hline Sugar-sweetened beverages and sweets & $82.02 \pm 7.55$ & $29.72 \pm 7.46$ & $14.37 \pm 7.20$ & $<0.001$ \\
\hline Red and processed meats & $54.98 \pm 2.81$ & $34.43 \pm 2.77$ & $24.92 \pm 2.68$ & $<0.001$ \\
\hline \multicolumn{5}{|l|}{ Nutrients } \\
\hline Sodium & $4205.86 \pm 122.32$ & $3326.08 \pm 120.78$ & $2865.86 \pm 116.58$ & $<0.001$ \\
\hline Energy & $2396.12 \pm 93.42$ & $2044.82 \pm 91.68$ & $2343.96 \pm 89.22$ & 0.01 \\
\hline Carbohydrate & $356.49 \pm 5.61$ & $370.58 \pm 5.54$ & $370.44 \pm 5.35$ & 0.12 \\
\hline Protein & $83.15 \pm 1.83$ & $84.14 \pm 1.80$ & $90.47 \pm 1.74$ & 0.008 \\
\hline Fat & $63.77 \pm 1.92$ & $58.47 \pm 1.89$ & $55.97 \pm 1.83$ & 0.01 \\
\hline Fiber & $25.83 \pm 0.81$ & $30.21 \pm 0.80$ & $33.54 \pm 0.77$ & $<0.001$ \\
\hline Calcium & $1223.35 \pm 43.88$ & $1332.90 \pm 43.33$ & $1449.38 \pm 41.82$ & 0.001 \\
\hline Pyridoxine & $2.46 \pm 0.13$ & $2.60 \pm 0.13$ & $2.74 \pm 0.12$ & 0.32 \\
\hline Folate & $486.42 \pm 10.94$ & $538.62 \pm 10.80$ & $599.84 \pm 10.42$ & $<0.001$ \\
\hline
\end{tabular}

Table 2. Dietary intakes of study participants by tertile categories of DASH Diet score. All values are mean \pm SE; energy intake is adjusted for age and sex, all other values are adjusted for age, sex and energy intake. ${ }^{\dagger}$ ANCOVA for all variables.

analyses (men: OR 2.29; 95\% CI 0.39-13.29 and women: 0.75; 95\% CI 0.23-2.45). Moreover, regarding EWGSOP1-sarcopenia, we failed to find any significant association between adherence to the DASH diet and sarcopenia either before (OR 0.73; 95\% CI 0.36-1.47) or after controlling for potential confounders (OR 0.78; 95\% 


\begin{tabular}{|c|c|c|c|c|}
\hline & \multicolumn{3}{|c|}{ Tertiles of DASH diet score } & \multirow[b]{2}{*}{$P^{\star}$} \\
\hline & $\mathrm{T}_{1}$ & $\mathrm{~T}_{2}$ & $T_{3}$ & \\
\hline DASH Score range & $<39$ & $39-49$ & $>49$ & \\
\hline \multicolumn{5}{|l|}{ Whole population } \\
\hline $\mathrm{n}$ & 96 & 98 & 106 & \\
\hline Muscle mass $\left[\mathrm{ASM} / \mathrm{h}^{2}\right](\mathrm{kg})$ & $6.71 \pm 1.01$ & $6.72 \pm 0.92$ & $6.40 \pm 1.01$ & 0.02 \\
\hline Hand grip strength (psi) & $11.82 \pm 3.74$ & $11.19 \pm 3.74$ & $10.20 \pm 3.07$ & 0.005 \\
\hline Gait speed $(\mathrm{m} / \mathrm{s})$ & $0.86 \pm 0.25$ & $0.82 \pm 0.20$ & $0.84 \pm 0.21$ & 0.55 \\
\hline Lower muscle mass $\mathrm{n}(\%)^{\dagger}$ & $38(39.6)$ & $31(31.6)$ & $39(36.8)$ & 0.50 \\
\hline Lower hand grip strength $\mathrm{n}(\%)^{\ddagger}$ & $25(26.0)$ & $31(31.6)$ & $40(37.7)$ & 0.20 \\
\hline Slower gait speed $(\mathrm{m} / \mathrm{s}) \mathrm{n}(\%)^{\S}$ & 38 (39.6) & $40(40.8)$ & $44(41.5)$ & 0.96 \\
\hline Sarcopenia n (\%)\| & $11(11.5)$ & $7(7.1)$ & $13(12.3)$ & 0.44 \\
\hline \multicolumn{5}{|l|}{ Men } \\
\hline $\mathrm{n}$ & 57 & 50 & 40 & \\
\hline Muscle mass $\left[\mathrm{ASM} / \mathrm{h}^{2}\right](\mathrm{kg})$ & $7.23 \pm 0.74$ & $7.20 \pm 0.70$ & $7.10 \pm 0.81$ & 0.69 \\
\hline Hand grip strength (psi) & $13.78 \pm 3.18$ & $13.88 \pm 2.94$ & $12.89 \pm 2.61$ & 0.23 \\
\hline Gait speed $(\mathrm{m} / \mathrm{s})$ & $0.91 \pm 0.21$ & $0.88 \pm 0.18$ & $0.87 \pm 0.22$ & 0.61 \\
\hline Lower muscle mass n (\%) & $25(43.9)$ & $21(42.0)$ & $19(47.5)$ & 0.87 \\
\hline Lower hand grip strength n (\%) & $9(15.8)$ & $9(18.0)$ & $7(17.5)$ & 0.95 \\
\hline Slower gait speed $(\mathrm{m} / \mathrm{s}) \mathrm{n}(\%)$ & $17(29.8)$ & $15(30.0)$ & $14(35.0)$ & 0.83 \\
\hline Sarcopenia n (\%) & $4(7.0)$ & $3(6.0)$ & $4(10.0)$ & 0.76 \\
\hline \multicolumn{5}{|l|}{ Women } \\
\hline $\mathrm{n}$ & 39 & 48 & 66 & \\
\hline Muscle mass $\left[\mathrm{ASM} / \mathrm{h}^{2}\right](\mathrm{kg})$ & $5.96 \pm 0.88$ & $6.23 \pm 0.85$ & $5.97 \pm 0.87$ & 0.23 \\
\hline Hand grip strength (psi) & $8.96 \pm 2.43$ & $8.40 \pm 2.04$ & $8.57 \pm 2.00$ & 0.46 \\
\hline Gait speed $(\mathrm{m} / \mathrm{s})$ & $0.79 \pm 0.28$ & $0.77 \pm 0.21$ & $0.82 \pm 0.20$ & 0.51 \\
\hline Lower muscle mass n (\%) & $13(33.3)$ & $10(20.8)$ & $20(30.3)$ & 0.37 \\
\hline Lower hand grip strength $\mathrm{n}(\%)$ & $16(41.0)$ & $22(45.8)$ & $33(50.0)$ & 0.66 \\
\hline Slower gait speed $(\mathrm{m} / \mathrm{s}) \mathrm{n}(\%)$ & $21(53.8)$ & $25(52.1)$ & 30 (45.5) & 0.65 \\
\hline Sarcopenia n (\%) & $7(17.9)$ & $4(8.3)$ & $9(13.6)$ & 0.41 \\
\hline
\end{tabular}

Table 3. Prevalence of sarcopenia and its components across tertile categories of DASH diet score, stratified by gender. ${ }^{*}$ Obtained from ANOVA for quantitative variables and chi-square for qualitative variables $(P<0.05$ significant). ${ }^{\dagger}$ Muscle mass lower than $5.5\left(\mathrm{~kg} / \mathrm{m}^{2}\right)$ for women and $7.0\left(\mathrm{~kg} / \mathrm{m}^{2}\right)$ for $\mathrm{men}^{38} .{ }^{\ddagger}$ Lower muscle strength were defined according previous study ${ }^{41}$. ${ }^{5}$ Gait speeds equal or slower than $0.8 \mathrm{~m} / \mathrm{s}^{38}$. ${ }^{\| S a r c o p e n i a}$ was defined based on European Working Group on Sarcopenia in Older People 2 (EWGSOP2) definition ${ }^{38}$.

CI 0.36-1.67). Such non-significant findings were also observed in the gender-stratified analyses (men: OR 1.22; 95\% CI 0.42-3.55 and women: 0.45; 95\% CI 0.14-1.44).

Linear regression analysis of the association between DASH diet score and components of sarcopenia are presented in Table 5. In the unadjusted model, the DASH diet score was inversely related to hand grip strength. However; after adjustment for potential confounders, this association became non-significant. No other overall association was seen between DASH diet score and other components of sarcopenia.

Multivariable-adjusted odds ratios for EWGSOP2-sarcopenia across tertile categories of DASH diet components are shown in Table 6. In terms of vegetables, when we controlled the analysis for potential confounding variables, participants in the highest tertile were 76\% less likely to have EWGSOP2-sarcopenia compared with those in the lowest tertile (OR $0.24 ; 95 \%$ CI $0.07-0.74$ ). No significant associations were seen between other components of DASH diet and odds of EWGSOP2-sarcopenia, after adjustment for potential confounders.

\section{Discussion}

We failed to find any significant association between adherence to the DASH diet and odds of sarcopenia after controlling for potential confounders. According to our knowledge, this is the first study from the Middle-Eastern countries that reports the association between adherence to the DASH diet and sarcopenia.

Considering increased age in the world, sarcopenia should be considered as an important public health problem $^{43}$. Dietary intakes can play a considerable role in maintaining muscle health and strength ${ }^{44}$. Several studies have shown that healthy dietary patterns were inversely associated with risk of sarcopenia ${ }^{45,46}$. In the present study, adherence to DASH diet was not significantly associated with odds of sarcopenia. Although no previous study has examined DASH diet in relation to sarcopenia, two recent studies have reported that adherence to a diet similar to the DASH diet is not associated with sarcopenia ${ }^{47,48}$. A large prospective cohort study by Chan et al. among Chinese men and women aged 65 years or older revealed no significant association between 


\begin{tabular}{|c|c|c|c|c|}
\hline & \multicolumn{3}{|c|}{ Tertiles of DASH diet score } & \multirow[b]{2}{*}{$P$ trend } \\
\hline & $\mathrm{T}_{1}$ & $T_{2}$ & $T_{3}$ & \\
\hline \multicolumn{5}{|c|}{ Sarcopenia $^{*}$} \\
\hline $\mathrm{n}$ & 96 & 98 & 106 & \\
\hline Crude & 1.00 & $0.59(0.22-1.60)$ & $1.08(0.45-2.54)$ & 0.82 \\
\hline Model $1^{\dagger}$ & 1.00 & $0.64(0.23-1.80)$ & $1.06(0.43-2.60)$ & 0.85 \\
\hline Model $2^{\ddagger}$ & 1.00 & $0.69(0.23-2.04)$ & $1.04(0.39-2.75)$ & 0.90 \\
\hline \multicolumn{5}{|l|}{ Men } \\
\hline $\mathrm{n}$ & 57 & 50 & 40 & \\
\hline Crude & 1.00 & $0.84(0.18-3.97)$ & $1.47(0.34-6.27)$ & 0.62 \\
\hline Model $1^{\S}$ & 1.00 & $0.83(0.17-4.07)$ & $1.71(0.38-7.60)$ & 0.50 \\
\hline Model 2 & 1.00 & $0.76(0.12-4.72)$ & $2.29(0.39-13.29)$ & 0.41 \\
\hline \multicolumn{5}{|l|}{ Women } \\
\hline $\mathrm{n}$ & 39 & 48 & 66 & \\
\hline Crude & 1.00 & $0.41(0.11-1.54)$ & $0.72(0.24-2.12)$ & 0.65 \\
\hline Model $1^{\S}$ & 1.00 & $0.51(0.13-1.97)$ & $0.79(0.26-2.40)$ & 0.77 \\
\hline Model 2 & 1.00 & $0.51(0.12-2.16)$ & $0.75(0.23-2.45)$ & 0.71 \\
\hline \multicolumn{5}{|c|}{ Sarcopeniall } \\
\hline $\mathrm{n}$ & 96 & 98 & 106 & \\
\hline Crude & 1.00 & $0.64(0.31-1.34)$ & $0.73(0.36-1.47)$ & 0.38 \\
\hline Model 1 & 1.00 & $0.66(0.31-1.40)$ & $0.75(0.36-1.55)$ & 0.43 \\
\hline Model 2 & 1.00 & $0.71(0.33-1.55)$ & $0.78(0.36-1.67)$ & 0.52 \\
\hline \multicolumn{5}{|l|}{ Men } \\
\hline $\mathrm{n}$ & 57 & 50 & 40 & \\
\hline Crude & 1.00 & $0.93(0.36-2.40)$ & $1.25(0.48-3.25)$ & 0.67 \\
\hline Model 1 & 1.00 & $0.90(0.34-2.37)$ & $1.06(0.39-2.86)$ & 0.92 \\
\hline Model 2 & 1.00 & $0.88(0.31-2.43)$ & $1.22(0.42-3.55)$ & 0.75 \\
\hline \multicolumn{5}{|l|}{ Women } \\
\hline $\mathrm{n}$ & 39 & 48 & 66 & \\
\hline Crude & 1.00 & $0.38(0.11-1.27)$ & $0.46(0.16-1.31)$ & 0.16 \\
\hline Model 1 & 1.00 & $0.41(0.12-1.38)$ & $0.46(0.16-1.34)$ & 0.18 \\
\hline Model 2 & 1.00 & $0.42(0.11-1.54)$ & $0.45(0.14-1.44)$ & 0.20 \\
\hline
\end{tabular}

Table 4. Multivariable-adjusted odds ratios (95\% CIs) for sarcopenia across tertile categories of DASH diet score, stratified by gender. ${ }^{\star}$ Sarcopenia was defined based on European Working Group on Sarcopenia in Older People 2 (EWGSOP2) definition ${ }^{38}$. ${ }^{\dagger}$ Model 1: Adjusted for age, sex and energy intake. ${ }^{\ddagger}$ Model 2: Further adjusted for physical activity, smoking, alcohol consumption, medication use (statin, ACEi, estrogen, testosterone), corticosteroid use and positive history of disease (asthma, arthritis, myocardial infarction, cerebrovascular accident, diabetes). ${ }^{\$}$ Model 1: Adjusted for age and energy intake. "Sarcopenia was defined based on European Working Group on Sarcopenia in Older People (EWGSOP) definition ${ }^{37}$.

\begin{tabular}{|c|c|c|c|c|c|c|c|c|c|c|c|c|}
\hline & \multicolumn{12}{|c|}{ DASH diet score } \\
\hline & \multicolumn{4}{|l|}{ Crude } & \multicolumn{4}{|c|}{ Model $1^{*}$} & \multicolumn{4}{|c|}{$\operatorname{Model} 2^{\dagger}$} \\
\hline & $\beta$ & $95 \% \mathrm{CI}$ & $P$ & $\mathbf{R}^{2}$ & $\beta$ & $95 \% \mathrm{CI}$ & $P$ & $\mathbf{R}^{2}$ & $\beta$ & 95\% CI & $P$ & $\mathbf{R}^{2}$ \\
\hline Muscle mass $\left[\mathrm{ASM} / \mathrm{h}^{2}\right](\mathrm{kg})$ & -0.006 & $-0.017,0.005$ & 0.29 & 0.004 & 0.002 & $-0.007,0.011$ & 0.67 & 0.340 & 0.001 & $-0.009,0.010$ & 0.94 & 0.354 \\
\hline Hand grip strength (psi) & -0.044 & $-0.083,-0.006$ & 0.02 & 0.017 & -0.006 & $-0.033,0.020$ & 0.62 & 0.571 & -0.003 & $-0.030,0.024$ & 0.82 & 0.588 \\
\hline Gait speed $(\mathrm{m} / \mathrm{s})$ & -0.001 & $-0.004,0.001$ & 0.33 & 0.003 & 0.001 & $-0.003,0.002$ & 0.85 & 0.112 & -0.001 & $-0.002,0.002$ & 0.96 & 0.162 \\
\hline
\end{tabular}

Table 5. Linear regression analysis of the association between DASH diet score and components of sarcopenia. ${ }^{\star}$ Model 1: Adjusted for age, sex and energy intake. ${ }^{\dagger}$ Model 2: Further adjusted for physical activity, smoking, alcohol consumption, medication use (statin, ACEi, estrogen, testosterone), corticosteroid use and positive history of disease (asthma, arthritis, myocardial infarction, cerebrovascular accident, diabetes).

Mediterranean Diet Score (MDS) and sarcopenia ${ }^{48}$. It should be noted that the MDS in Chan et al.'s study was based on 6 healthy components (vegetables, legumes, fruits and nuts, cereal, fish and mono-unsaturated to saturated lipids ratio) and 3 unhealthy components (meat, poultry and dairy products), which is different from the DASH diet scoring in our study. In addition, studying various populations with different dietary patterns may provide better insight into the association between adherence to a healthy dietary pattern and risk of developing 


\begin{tabular}{|c|c|c|c|c|}
\hline & \multicolumn{3}{|c|}{ Tertiles of components of the DASH diet } & \multirow[b]{2}{*}{$P$ trend } \\
\hline & $\mathrm{T}_{1}$ & $\mathbf{T}_{2}$ & $\mathbf{T}_{3}$ & \\
\hline Whole-grains & 1.00 & $1.05(0.36-3.00)$ & $1.57(0.59-4.15)$ & 0.35 \\
\hline Fruit & 1.00 & $0.61(0.22-1.66)$ & $0.80(0.31-2.07)$ & 0.65 \\
\hline Vegetables & 1.00 & $0.57(0.22-1.48)$ & $0.24(0.07-0.74)$ & 0.01 \\
\hline Low-fat dairy products & 1.00 & $2.50(0.83-7.49)$ & $1.88(0.61-5.75)$ & 0.34 \\
\hline Nuts, legumes and soy & 1.00 & $0.36(0.11-1.10)$ & $0.75(0.30-1.85)$ & 0.51 \\
\hline Red and processed meats & 1.00 & $1.10(0.40-3.06)$ & $1.19(0.44-3.19)$ & 0.73 \\
\hline Sodium & 1.00 & $2.36(0.87-6.36)$ & $0.65(0.19-2.18)$ & 0.46 \\
\hline Sweats and sweetened beverages & 1.00 & $2.11(0.71-6.19)$ & $1.59(0.54-4.62)$ & 0.43 \\
\hline
\end{tabular}

Table 6. The association between components of the Dietary Approaches to Stop Hypertension (DASH) diet and sarcopenia ${ }^{\star}$ (Odds ratios and 95\% confidence intervals) ${ }^{\star}{ }^{\star}$ Sarcopenia was defined based on European Working Group on Sarcopenia in Older People 2 (EWGSOP2) definition ${ }^{38}$. Binary logistic regression was used to obtain OR and 95\% CI. The overall trend of the OR across increasing tertiles was examined by considering the median score in each category as a continuous variable. ${ }^{\dagger}$ ORs are adjusted for age, sex, energy intake, physical activity, smoking, alcohol consumption, medication use (statin, ACEi, estrogen, testosterone), corticosteroid use and positive history of disease (asthma, arthritis, myocardial infarction, cerebrovascular accident, diabetes).

sarcopenia. Findings from another prospective study on 100 outpatients aged 18 or more, with a recent diagnosis of GI cancer, revealed that adherence to a diet rich in legumes, vegetables and fruit was not significantly associated with sarcopenia ${ }^{47}$. However, it must be kept in mind that this study has been done on patients with cancer, in which cytokines can lead to severe muscle wasting (sarcopenia) and cachexia ${ }^{49}$. Therefore, this pattern of sarcopenia might be different from the one we studied in the current study and their findings cannot easily be generalized to other healthy populations. Unlike our findings, a cross-sectional study indicated a favorable association between adherence to the DASH diet and handgrip strength, as a component of sarcopenia ${ }^{32}$. In a quasi-experimental study, combined resistance training (RT) along with a counseling on modified DASH diet in 95 overweight and obese older adults aged 55 to 80 years resulted in significant improvements in muscle strength, as measured by handgrip strength and knee extensor torque ${ }^{33}$. Lack of any significant association between adherence to DASH-style diet and sarcopenia might be partially explained by the low number of sarcopenic patients in our study. When we categorized participants based on tertiles of DASH diet score, only 18 participants in the top tertile had sarcopenia. Such a low number of patients would result in a very wide range of confidence intervals, which can hide the probable association of DASH diet and sarcopenia. In addition, we used the squeeze bulb dynamometer for hand grip strength instead of the Jamar handheld dynamometer, which has been accepted as the gold standard ${ }^{50}$. Therefore, some sort of misclassifications of study participants in terms of sarcopenia cannot be ignored. Further studies with a large sample of sarcopenic patients might be required to shed light on this association.

We did not find any significant association between adherence to DASH diet and odds of sarcopenia. However, there are some probable mechanisms that can help explaining the association between DASH and sarcopenia. Consumption of DASH diet results in improving poor nutritional status, suppressing inflammation process ${ }^{51}$, reducing oxidative stress as well as neutralizing mild metabolic acidosis ${ }^{16,52-54}$. These pathways may help preventing age-related muscle mass and strength decline.

This study has several strengths. This is the first report examining the association between adherence to the DASH diet and risk of sarcopenia. We controlled for a wide range of confounders in the current analysis. In addition, we used a validated FFQ for assessment of dietary intake. Our study has some limitations. The major one is its cross-sectional design, which precludes us inferring causal associations. Thus, our findings need to be confirmed in prospective studies. Due to the use of the FFQ, misclassification of study participants and measurement error are another concerns. In addition, the FFQ could not provide precise measurement of sodium intake while it is a major component of the DASH diet. Also, we did not collect 24 -h urine samples of participants to assess sodium intake. Moreover, due to financial constraints as well as limited access to the only available DEXA device in Tehran (maximum 300 cases), this study was done on a small sample from the confined region where the device was located. Therefore, caution is needed when generalizing the results to the whole Iranian population. Further, we had only collected information on arthritis among musculoskeletal diseases. No information was collected about other musculoskeletal disease and anti-psychotic drugs in the current study. Finally, our study consisted of a relative young population including 300 subjects aged $\geq 55$ years, which can explain the low prevalence of sarcopenic subjects in the sample enrolled.

In conclusion, we found that adherence to the DASH diet was not significantly associated with odds of sarcopenia after controlling for confounders. Future prospective studies are required to confirm these findings.

Received: 10 February 2020; Accepted: 28 October 2020

Published online: 09 November 2020 


\section{References}

1. Cruz-Jentoft, A. J., Landi, F., Topinkova, E. \& Michel, J.-P. Understanding sarcopenia as a geriatric syndrome. Curr. Opin. Clin. Nutr. Metab. Care 13, 1-7 (2010).

2. Morley, J. E., Baumgartner, R. N., Roubenoff, R., Mayer, J. \& Nair, K. S. Sarcopenia. J. Lab. Clin. Med. 137, 231-243 (2001).

3. Delmonico, M. J. et al. Alternative definitions of sarcopenia, lower extremity performance, and functional impairment with aging in older men and women. J. Am. Geriatr. Soc. 55, 769-774 (2007).

4. Goodpaster, B. H. et al. The loss of skeletal muscle strength, mass, and quality in older adults: the health, aging and body composition study. J. Gerontol. Ser. A Biol. Sci. Med. Sci. 61, 1059-1064 (2006).

5. Vellas, B. et al. Implications of ICD-10 for sarcopenia clinical practice and clinical trials: report by the International Conference on Frailty and Sarcopenia Research Task Force. J. Frailty Aging 7, 2-9 (2018).

6. Janssen, I., Shepard, D. S., Katzmarzyk, P. T. \& Roubenoff, R. The healthcare costs of sarcopenia in the United States. J. Am. Geriatr. Soc. 52, 80-85 (2004).

7. Kim, H. et al. Sarcopenia: prevalence and associated factors based on different suggested definitions in community-dwelling older adults. Geriatr. Gerontol. Int. 16, 110-122 (2016).

8. Mayhew, A. et al. The prevalence of sarcopenia in community-dwelling older adults, an exploration of differences between studies and within definitions: a systematic review and meta-analyses. Age Ageing 48, 48-56 (2018).

9. Johnson, A. E. et al. Fruit and vegetable consumption in later life. Age Ageing 27, 723-728 (1998).

10. Margetts, B., Thompson, R., Elia, M. \& Jackson, A. Prevalence of risk of undernutrition is associated with poor health status in older people in the UK. Eur. J. Clin. Nutr. 57, 69 (2003).

11. Svetkey, L. P. et al. The DASH diet, sodium intake and blood pressure trial (DASH-sodium): rationale and design. J. Am. Diet. Assoc. 99, S96-S104 (1999).

12. Salehi-Abargouei, A., Maghsoudi, Z., Shirani, F. \& Azadbakht, L. Effects of Dietary Approaches to Stop Hypertension (DASH)-style diet on fatal or nonfatal cardiovascular diseases-incidence: a systematic review and meta-analysis on observational prospective studies. Nutrition 29, 611-618 (2013).

13. Onvani, S., Haghighatdoost, F. \& Azadbakht, L. Dietary approach to stop hypertension (DASH): diet components may be related to lower prevalence of different kinds of cancer: a review on the related documents. J. Res. Med. Sci. 20, 707 (2015).

14. Jannasch, F., Kröger, J. \& Schulze, M. B. Dietary patterns and type 2 diabetes: a systematic literature review and meta-analysis of prospective studies. J. Nutr. 147, 1174-1182 (2017).

15. Kim, J., Lee, Y., Kye, S., Chung, Y.-S. \& Kim, K.-M. Association of vegetables and fruits consumption with sarcopenia in older adults: the Fourth Korea National Health and Nutrition Examination Survey. Age Ageing 44, 96-102 (2015).

16. Dawson-Hughes, B., Harris, S. S. \& Ceglia, L. Alkaline diets favor lean tissue mass in older adults. Am. J. Clin. Nutr. 87, 662-665 (2008).

17. Neville, C. E. et al. Effect of increased fruit and vegetable consumption on physical function and muscle strength in older adults. Age 35, 2409-2422 (2013).

18. Lauretani, F. et al. Low plasma carotenoids and skeletal muscle strength decline over 6 years. J. Gerontol. Ser. A Biol. Sci. Med. Sci. 63, 376-383 (2008).

19. Radavelli-Bagatini, S., Zhu, K., Lewis, J. R. \& Prince, R. L. Dairy food intake, peripheral bone structure, and muscle mass in elderly ambulatory women. J. Bone Miner. Res. 29, 1691-1700 (2014).

20. Lopes, H. F. et al. DASH diet lowers blood pressure and lipid-induced oxidative stress in obesity. Hypertension 41, 422-430 (2003).

21. Asemi, Z., Samimi, M., Tabassi, Z., Sabihi, S.-S. \& Esmaillzadeh, A. A randomized controlled clinical trial investigating the effect of DASH diet on insulin resistance, inflammation, and oxidative stress in gestational diabetes. Nutrition 29, 619-624 (2013).

22. Meng, S.-J. \& Yu, L.-J. Oxidative stress, molecular inflammation and sarcopenia. Int. J. Mol. Sci. 11, 1509-1526 (2010).

23. Baumann, C. W., Kwak, D., Liu, H. M. \& Thompson, L. V. Age-induced oxidative stress: how does it influence skeletal muscle quantity and quality?. J. Appl. Physiol. 121, 1047-1052 (2016).

24. Batsis, J. A., Mackenzie, T. A., Jones, J. D., Lopez-Jimenez, F. \& Bartels, S. J. Sarcopenia, sarcopenic obesity and inflammation: results from the 1999-2004 National Health and Nutrition Examination Survey. Clin. Nutr. 35, 1472-1483 (2016).

25. Beyer, I., Mets, T. \& Bautmans, I. Chronic low-grade inflammation and age-related sarcopenia. Curr. Opin. Clin. Nutr. Metab. Care 15, 12-22 (2012).

26. Cleasby, M. E., Jamieson, P. M. \& Atherton, P. J. Insulin resistance and sarcopenia: mechanistic links between common comorbidities. J. Endocrinol. 229, R67-R81 (2016).

27. Boccardi, V. \& Paolisso, G. The link between insulin resistance and mobility limitation in older persons. Curr. Pharm. Des. 20, 3095-3098 (2014).

28. Ali Mohsenpour, M. et al. Adherence to Dietary Approaches to Stop Hypertension (DASH)-style diet and the risk of cancer: a systematic review and meta-analysis of cohort studies. J. Am. Coll. Nutr. 38, 513-525 (2019).

29. Kim, T. N. \& Choi, K. M. The implications of sarcopenia and sarcopenic obesity on cardiometabolic disease. J. Cell. Biochem. 116, $1171-1178(2015)$

30. Scott, D., de Courten, B. \& Ebeling, P. R. Sarcopenia: a potential cause and consequence of type 2 diabetes in Australia's ageing population?. Med. J. Aust. 205, 329-333 (2016).

31. Fearon, K. et al. Definition and classification of cancer cachexia: an international consensus. Lancet Oncol. 12, 489-495 (2011).

32. Kuczmarski, M. F., Pohlig, R., Shupe, E. S., Zonderman, A. \& Evans, M. Dietary protein intake and overall diet quality are associated with handgrip strength in African American and white adults. J. Nutr. Health Aging 22, 1-10 (2018).

33. Straight, C. R. et al. Effects of resistance training and dietary changes on physical function and body composition in overweight and obese older adults. J. Phys. Act. Health 9, 875-883 (2012).

34. Hashemi, R. et al. Sarcopenia and its determinants among Iranian elderly (SARIR): study protocol. J. Diabetes Metab. Disord. 11, 23 (2012).

35. Mirmiran, P., Esfahani, F. H., Mehrabi, Y., Hedayati, M. \& Azizi, F. Reliability and relative validity of an FFQ for nutrients in the Tehran lipid and glucose study. Public Health Nutr. 13, 654-662 (2010).

36. Willett, W. C., Howe, G. R. \& Kushi, L. H. Adjustment for total energy intake in epidemiologic studies. Am. J. Clin. Nutr. 65, 1220S-1228S (1997).

37. Cruz-Jentoft, A. J. et al. Sarcopenia: European consensus on definition and diagnosis Report of the European Working Group on Sarcopenia in Older PeopleA J. Cruz-Gentoft et al. Age Ageing 39, 412-423 (2010).

38. Cruz-Jentoft, A. J. et al. Sarcopenia: revised European consensus on definition and diagnosis. Age Ageing 48, 16-31 (2019).

39. Heymsfield, S. B. et al. Appendicular skeletal muscle mass: measurement by dual-photon absorptiometry. Am. J. Clin. Nutr. 52, 214-218 (1990).

40. Richards, L. \& Palmiter-Thomas, P. Grip strength measurement: a critical review of tools, methods, and clinical utility. Crit. Rev. Phys. Rehabil. Med. 8, 87-109 (1996).

41. Merkies, I. et al. Assessing grip strength in healthy individuals and patients with immune-mediated polyneuropathies. Muscle Nerve 23, 1393-1401 (2000).

42. Committee, I. R. Guidelines for Data Processing and Analysis of the International Physical Activity Questionnaire (IPAQ)-Short and Long Forms. https://www.ipaq.ki.se/scoring.pdf. (2005). 
43. United Nations, D. o. E. \& Affairs, S. (United Nations New York, 2017).

44. Granic, A., Sayer, A. A. \& Robinson, S. M. Dietary patterns, skeletal muscle health, and sarcopenia in older adults. Nutrients 11, 745 (2019).

45. Mohseni, R. et al. Relationship between major dietary patterns and sarcopenia among menopausal women. Aging Clin. Exp. Res. 29, 1241-1248 (2017).

46. Kim, J., Lee, Y., Kye, S., Chung, Y. S. \& Kim, K. M. Association between healthy diet and exercise and greater muscle mass in older adults. J. Am. Geriatr. Soc. 63, 886-892 (2015).

47. Velho, S. et al. Dietary patterns and its relationship to sarcopenia in Portuguese patients with gastrointestinal cancer: an exploratory study. Nutrition 63-64, 193-199 (2019).

48. Chan, R., Leung, J. \& Woo, J. A prospective cohort study to examine the association between dietary patterns and sarcopenia in Chinese community-dwelling older people in Hong Kong. J. Am. Med. Dir. Assoc. 17, 336-342 (2016).

49. Argilés, J. M., Busquets, S., Felipe, A. \& López-Soriano, F. J. Molecular mechanisms involved in muscle wasting in cancer and ageing: cachexia versus sarcopenia. Int. J. Biochem. Cell Biol. 37, 1084-1104 (2005).

50. Roberts, H. C. et al. A review of the measurement of grip strength in clinical and epidemiological studies: towards a standardised approach. Age Ageing 40, 423-429 (2011).

51. Soltani, S., Chitsazi, M. J. \& Salehi-Abargouei, A. The effect of dietary approaches to stop hypertension (DASH) on serum inflammatory markers: a systematic review and meta-analysis of randomized trials. Clin. Nutr. 37, 542-550 (2018).

52. Manchali, S., Murthy, K. N. C. \& Patil, B. S. Crucial facts about health benefits of popular cruciferous vegetables. J. Funct. Foods 4, 94-106 (2012)

53. Frei, B., England, L. \& Ames, B. N. Ascorbate is an outstanding antioxidant in human blood plasma. Proc. Natl. Acad. Sci. 86, 6377-6381 (1989).

54. Rose, R. \& Bode, A. Biology of free radical scavengers: an evaluation of ascorbate. FASEB J. 7, 1135-1142 (1993).

\section{Acknowledgements}

We wish to thank all individuals who kindly participated in our study.

\section{Author contributions}

S.S., R.H., R.H., A.D.M. and A.E. contributed to the conception, design, data collection, statistical analyses, data interpretation, manuscript drafting, approval of the final version of the manuscript and agreed for all aspects of the work.

\section{Funding}

The financial support for this study comes from the Tehran Endocrine and Metabolism Research Center and the Tehran University of Medical Science.

\section{Competing interests}

The authors declare no competing interests.

\section{Additional information}

Correspondence and requests for materials should be addressed to R.H. or A.E.

Reprints and permissions information is available at www.nature.com/reprints.

Publisher's note Springer Nature remains neutral with regard to jurisdictional claims in published maps and institutional affiliations.

(c) (i) Open Access This article is licensed under a Creative Commons Attribution 4.0 International License, which permits use, sharing, adaptation, distribution and reproduction in any medium or format, as long as you give appropriate credit to the original author(s) and the source, provide a link to the Creative Commons licence, and indicate if changes were made. The images or other third party material in this article are included in the article's Creative Commons licence, unless indicated otherwise in a credit line to the material. If material is not included in the article's Creative Commons licence and your intended use is not permitted by statutory regulation or exceeds the permitted use, you will need to obtain permission directly from the copyright holder. To view a copy of this licence, visit http://creativecommons.org/licenses/by/4.0/.

(c) The Author(s) 2020 